\title{
Decision Support System for Plant and Crop Treatment and Protection Based on Wireless Sensor Networks
}

\author{
Zoran Stamenković( ${ }^{1)}$, Siniša Randjićc ${ }^{2)}$, Ignacio Santamaria ${ }^{3)}$, Dušan Marković ${ }^{2}$, Steven Van \\ Vaerenbergh $^{3)}$, and Uroš Pešović ${ }^{2)}$ \\ 1) IHP, Frankfurt (Oder), Germany \\ 2) University of Kragujevac, Kragujevac, Serbia \\ 3) University of Cantabria, Santander, Spain \\ stamenko@ihp-microelectronics.com
}

\begin{abstract}
A decision support system (DSS) able to control the course of agricultural production and protect healthy and treat diseased plants and crops taking into account the temporal and spatial variability of environmental parameters has been described. It is based on remote sensing and the most sophisticated machine learning techniques: Gaussian Processes (GPs) and Deep Neural Networks (DNNs). An example of knowledge extraction and actionable rule definition has been presented too.
\end{abstract}

\section{INTRODUCTION}

Wireless sensor networks (WSN) have been extensively applied in agriculture [1]-[5]. Thanks to the deployment of sensor nodes on a large area, farmers can receive comprehensive information on the condition of environment and crops. At the same time, the data is obtained in real time and the decisions are made on the basis of real field measurements, not statistical values (Figure 1). By measuring critical parameters like temperature, humidity, insolation, moisture, carbon-dioxide or nitrogen concentration, or detecting economically important pests, farmers are able to anticipate plant health risks, and, when necessary to follow a suitable course of action, including timely pesticide treatment.

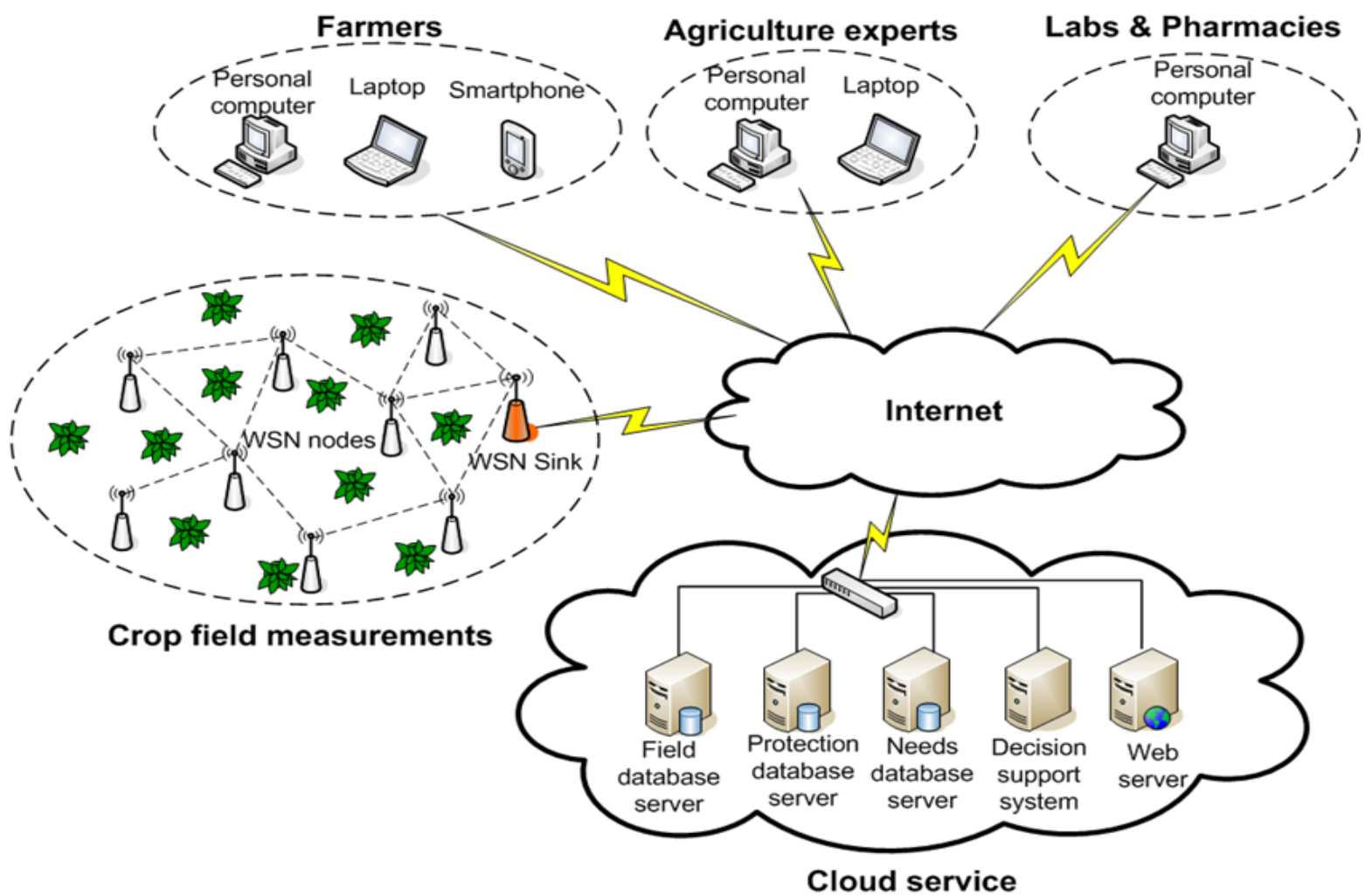

Fig. 1. WSN based information system for agricultural applications. 
Gathered data of deployed WSN is transmitted by a WSN gateway to a central computer which can store, analyze, and, if necessary, process it. By linking the central computer to the Internet (by a Web server or cloud system), the processed data is accepted and distributed to concerned parties (farmers, technicians, specialists, manufacturers, and retailers). This data is used to increase the yield and quality of feed and food.

On the basis of the information contained within a WSN, it is possible to develop a decision support system which can affect the course of agricultural production and help to protect healthy and treat diseased plants and crops, in particular, taking into account the temporal and spatial variability of environmental parameters.

The next section gives a brief overview of the wireless sensor networks and nodes for agricultural applications. Section 3 describes the proposed decision support system based on smart remote sensing, knowledge extraction, and timely defined actionable rules. Finally, the conclusions are drawn in Section 4.

\section{WIRELESS SENSOR NETWORKS}

Structure of an agro-meteorological sensor network based on sensor nodes and a network gateway to the Internet [6] is presented in Figure 1. In the field of plant/crop monitoring, wireless sensors have been developed to collect data on the air, soil, and leaf temperature, chlorophyll content, and plant water status. Based on these data, farmers are able to detect problems at an early stage and implement real-time solutions. The health and moisture of soil is a basic prerequisite for efficient plant and crop cultivation. Sensors contribute to real-time monitoring of variables such as soil fertility, soil water availability and soil compaction. Further, sensor nodes communicate with radio or weather stations and provide climate and micro-climate data.

Sensors registering temperature and relative humidity can contribute to detect conditions under which disease infestation is likely to occur. Microphone sensors can be used to detect distinct sound patterns, produced by specific kind of insects. In addition, camera sensors can be used to take pictures of plant habitus to observe changes caused by bacteria, viruses, fungus or pheromone traps which are used to lure insects. Camera sensors can provide a detailed history, which enables tracking dynamics of pest outbreak.
Sensors are compact, highly-accurate, powerefficient, and reliable electrical devices that have to be integrated with other electronic components in a sensor node (Figure 2). A wireless sensor node is equipped with a microcontroller, a radio device, and a number of dedicated sensors. The complete system is powered on with a rechargeable battery. Recharging is provided by some of the energy harvesting devices, usually, solar panels or wind mill generators.

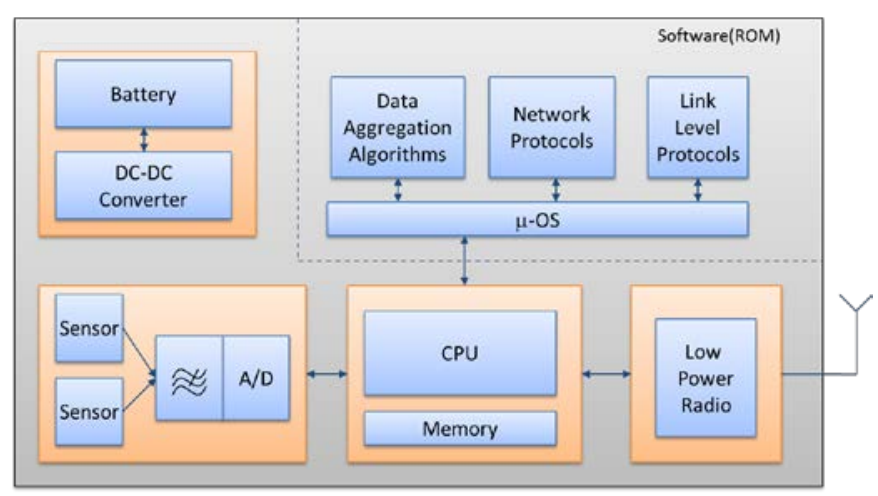

Fig. 2. Architecture of a wireless sensor node.

As sensor nodes in agriculture applications are deployed in thousands, they have to meet the following requirements:

- High readiness as a maturity for field deployment in terms of economic and engineering efficiency,

- High scalability to distributed agricultural monitoring tasks and, consequently, small size and low price to scale up to many distributed systems.

These nodes can communicate with a gateway, which has the capability of communicating with other networks, such as Wireless Local Area Networks (WLAN), Wireless Personal Area Networks (WPAN), 3G/4G cellular broadband systems or, in the near future, $5 G$ networks. Wireless sensor nodes, available on the market, include temperature sensors, humidity sensors, barometric pressure sensors, soil moisture sensors, soil temperature sensors, wind speed sensors, rainfall meters, light sensors, and solar radiation sensors. They can use various wireless standards to communicate among themselves.

Besides its sensing functionality, each wireless sensor node has communication functionality implemented in form of a network protocol. Network protocols are designed as a layered structure (protocol stack) in which each layer has specific functionalities. 
The layered structure of network protocols enables that the same upper layer services can be provided, independently of the functionality of lower layers. The main design goal for network protocols for WSN is to increase the node's energy efficiency in order to prolong the node's lifetime. Besides the use of energy efficient hardware, the energy can be saved using MAC (Media Access Control) protocols, which enable reliable communication with long periods of inactivity and exploit low power modes of the device. These modes increase the energy efficiency but create problems in the exchange of information.

WLAN is a flexible data communication protocol implemented to extend a wired local area network, such as the Ethernet. Its bandwidth is 11 Mbit and it operates at $2.4 \mathrm{GHz}$ frequency. Bluetooth is a wireless protocol that is used for short-range communication. It uses the $2.4 \mathrm{GHz}$ radio band to communicate at $1 \mathrm{Mbit}$ between up to eight devices. Bluetooth is considered a cable replacement for mobile devices. It is mainly designed to maximize the ad-hoc networking. ZigBee is another promising communication protocol for wireless sensor networks. It adds network, security, and user software to the IEEE 802.15.4 physical radio providing low data rate connectivity among relatively simple devices that consume little energy and connect over short distances.
WSN nodes include a number of hardware resources that are usually allocated in an orderly and controlled manner by node's operating system (OS) [7]. The OS enables easier development of target applications, since the application programmer can invoke already implemented OS services through system calls.

\section{DECISION SUPPORT SYSTEM}

An advanced decision support system (DSS) that assists farmers in making data-driven and rule-based decisions has been developed. The proposed rulebased decision support system concept is depicted in Figure 3. It merges information coming from the features, variables, and correlations extracted by the machine learning algorithms and previous knowledge in the existing databases. The final goal is to learn actionable rules that assist the farmer and help the decision-making process.

First, images and data are collected and preprocessed by a WSN. Then, the knowledge extraction algorithms which identify the key dependencies in observed data are applied. Finally, actionable rules which facilitate the protection of healthy and the treatment of diseased plants/crops are learned.

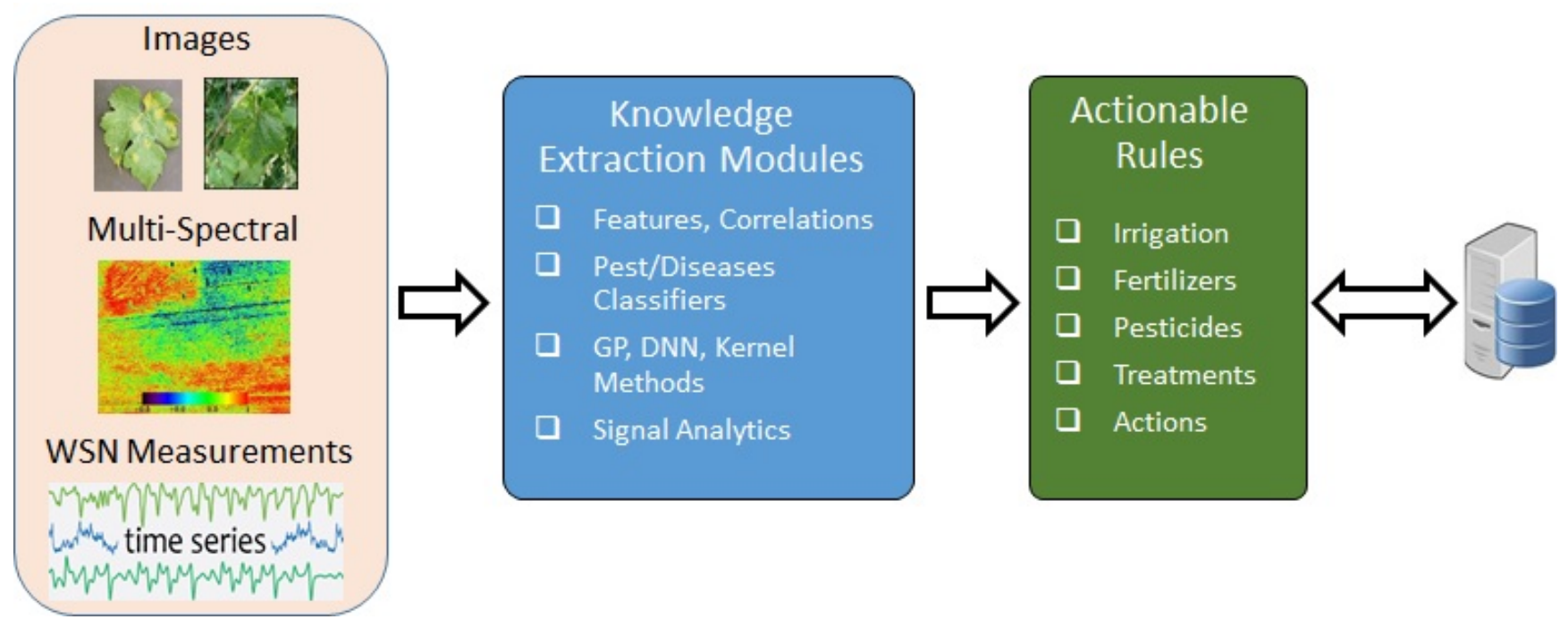

Fig. 3. Decision support system concept.

\subsection{Images and Sensor Measurements}

One of the decisions to be taken is to determine the right time to take action in the fight against pests. A monitoring system of pheromone traps for insects is presented in [8]. The main part of the system is a sensor device that measures temperature, relative humidity, and is also equipped with a small camera that takes images of sticky pheromone traps. Measured data whit captured images could be transferred to remote server for presentation, storage and further analysis. Part of the analysis can be done on the sensor 
side while a more complex analysis and prediction can be performed on remote servers on cloud. The basic idea is to analyze variations on images of the traps with caught pests in a longer period of time and provide information on their significant changes. A photo of trap insects on sticky surface, that would be processing, was shown in Figure 4.

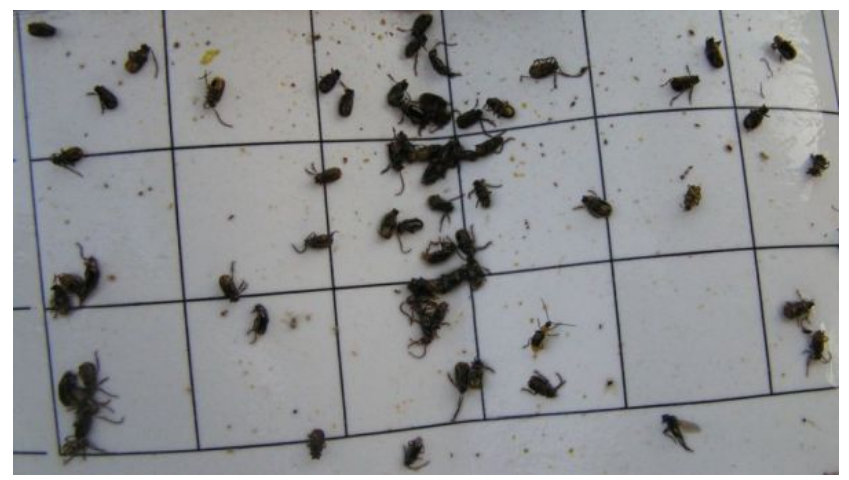

Fig. 4. Sticky surface in pheromone traps for pests.

Counting insects that are coughed on traps is not a problem when the number of insects is small, but as their number significantly increases, they usually stuck one to the other. Also, they lose their color and the identification by color tone is not reliable (the insects become visually dark stains). To solve the problem, a simple solution has been used: just the image portion occupied by black or near black segments has been considered. Knowing the size of an average individual insect, the total number of insects can be simply calculated taking into account all osculated image segments. For the sake of comparison, the numbers of caught insects counted visually and calculated by image analysis are shown in Table 1.

Table 1. Visually counted and calculated number of insects

\begin{tabular}{ccc}
\hline \hline Date & $\begin{array}{c}\text { Counted number } \\
\text { of insects }\end{array}$ & $\begin{array}{c}\text { Calculated } \\
\text { number of insects }\end{array}$ \\
\hline $11 / 07 / 2017$ & 2 & 2 \\
\hline $20 / 07 / 2017$ & 66 & 67.3 \\
\hline $02 / 08 / 2017$ & 6 & 6 \\
\hline $15 / 08 / 2017$ & 76 & 73.5 \\
\hline
\end{tabular}

The main benefit for users is to get information about significant changes of the number of insects in the field. Introduced solution is a part of the proposed decision support system. The idea is to provide the upto-date measurements (number of caught insects) and to conduct the prediction based on the previous measurements of this and other correlated parameters. It provides an essential support to farmers eliminating the need for visit of all agricultural fields to get the relevant information about pest emergence in time and take action on their suppression.

Sensor measurements can provide the values of various parameters like the air temperature, relative humidity, air pressure, soil temperature, insolation, rainfall, and others. This information can be used to assess whether certain crops are suitable for planting in a given location. Also, it can help to determine the period of time when there is a significant risk of pest and/or plant disease outbreak.

\subsection{Knowledge Extraction}

A sensor node is capable of a limited amount of signal processing, being typically restricted to the conditioning, filtering, simple distributed averaging, and detection. Then, these filtered or averaged measurements are wirelessly transmitted to a central entity where more sophisticated machine learning techniques can be applied to infer relevant agrometeorological parameters.

\section{Gaussian Processes}

Over the past two decades, the application of data processing and machine learning techniques in agriculture and crop protection has evolved rapidly along two main directions: a) the deployment of more and more sensors providing multiple sources of information, which goes beyond collecting just environmental and climate data [9], [10] and b) the use of more advanced machine learning techniques [11], [12]. Gaussian Processes (GPs) [11] are Bayesian tools for discriminative machine learning (for example, regression, classification, and dimensionality reduction). GPs can be interpreted as a family of Kernel Methods with the additional advantage of providing a full conditional statistical description for the predicted variable (for example, soil moisture or nitrogen concentration), which can be used primarily to establish confidence intervals and to set the method parameters (called hyper-parameters). In short, GPs assume that a GP prior distribution governs the set of possible latent functions (which are unobserved) and the likelihood (of the latent function) and observations shape this prior to produce the posterior probabilistic estimates. Consequently, the joint distribution of training and test data is a multidimensional Gaussian Process and the predicted distribution is estimated by conditioning on the training data.

Figure 5 presents a series of hourly dry-bulb temperature measurements depicted as blue dots. A 
GP trained on these measurements is capable of inferring missing values (middle of the time series) and predicting future readings several time steps ahead (right side of the time series). The predicted values are shown in red. The 95\% confidence interval is shown in gray.

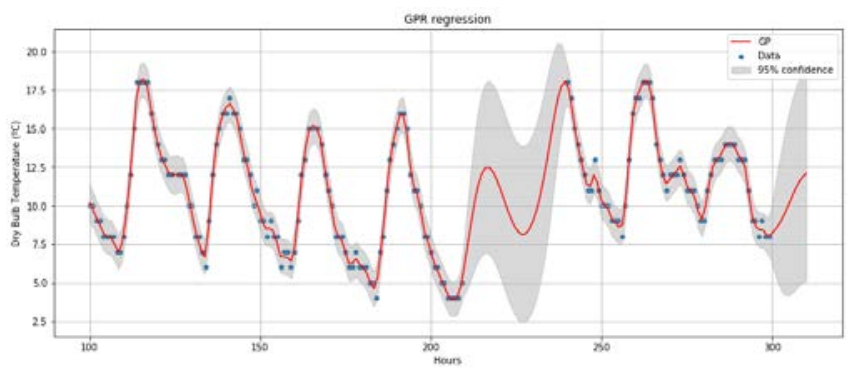

Fig. 5. Prediction of a temperature time series from sensor measurements using GPs.

Figure 6 shows a set of humidity sensors (depicted as blue dots) providing sparse noisy measurements used as inputs of a GP-based interpolation process that reconstructs the $2 \mathrm{D}$ spatial humidity field.
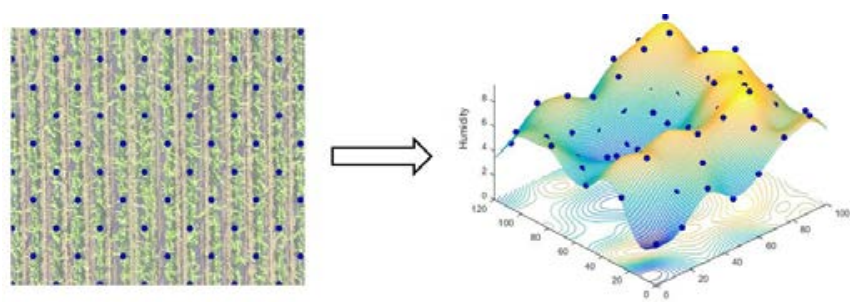

Fig. 6. Reconstruction of a 2D humidity field from sensor measurements using GPs.

\section{Deep Neural Networks}

A major recent breakthrough in artificial intelligence has been the concept of deep learning and especially Deep Neural Networks (DNNs), which have achieved impressive success in solving hard challenges in many fields including computer vision and image processing [13]. Since images taken from cameras and multispectral images taken from drones are key components of the proposed DSS, DNNs will be the most important component of the knowledge extraction process. Deep Neural Networks use a cascade of layers of simple nonlinear processing units (or linear convolutions) for feature extraction and transformation, where each layer uses the output from the previous layer as input. Figure 7 shows an example of a DNN setup.

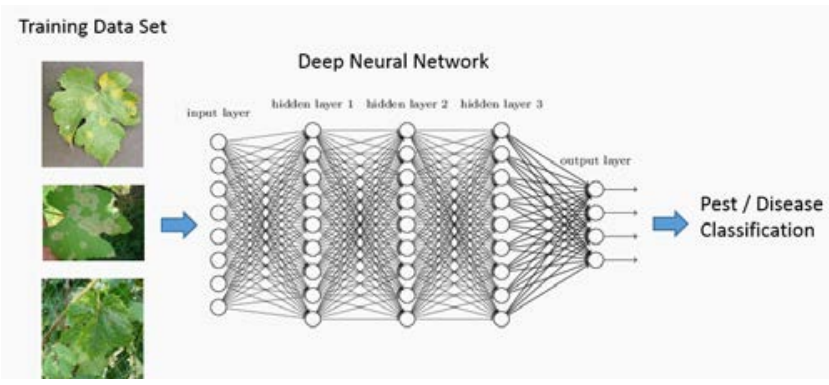

Fig. 7. Architecture of a Deep Neural Network.

A DNN takes an image at its input layer and transforms this image through its intermediate layers until a classification label is obtained at its output layer. The internal coefficients of the DNN are adjusted during the training phase, which aims to minimize the average output error obtained for a set of training images. Figure 8 illustrates the training phase for a DNN trained on a plant recognition task. The blue curve indicates the average error on the training data, along several passes (or epochs) over the entire training set. The red curve shows the average error the DNN obtains on a separate set of data that was not used for training.

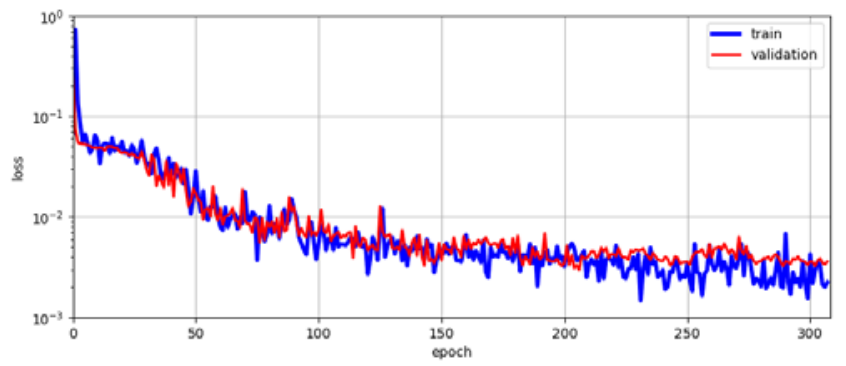

Fig. 8. Learning curves of a Deep Neural Network.

Although the fundamental principles behind DNNs are still under discussion, there is consensus that DNNs essentially establish a hierarchical and distributed representation of data, by means of which hidden data invariances are revealed. Another important ingredient for the successful application of DNNs is the availability of large amounts of unlabelled data, which allows the unsupervised learning of the multiple representations of data in hidden layers. Although the application of DNNs in precision agriculture is still largely unexplored, some recent work has also shown significant improvement in the classification of plant diseases based on images of plant leaves [14]. 


\subsection{An Example of Defining Actionable Rules}

Actionable rules provide the farmers explicit suggestions of actions to improve the treatment and protection of plants/crops. To define the actionable rules, a careful selection of relevant environmental, biological, and physiological attributes of a given crop type has been carried out. Afterwards, the field sensor measurements and data collection for 836 instances of the kidney-bean plant have been done. The attributes and their values are presented in Table 2.

Table 2. Relevant attributes and their values for a kidneybean plantation in Southern Serbia

\begin{tabular}{cc}
\hline \hline Attribute & Value \\
\hline Time Period & April-October \\
\hline Precipitations & Low, Normal, High \\
\hline Temperature & Low, Normal, High \\
\hline $\begin{array}{c}\text { Damaged } \\
\text { Area }\end{array}$ & $\begin{array}{c}\text { Scattered, Lower Parts, Upper Parts, } \\
\text { Whole Field }\end{array}$ \\
\hline Severity & Minor, Normal, Severe \\
\hline Seed & Treated, Non-Treated (Fungicide) \\
\hline Plant Growth & Normal, Abnormal \\
\hline Leaves & Normal, Abnormal \\
\hline Leaf Mildew & Absent, Lower Surface, Upper Surface \\
\hline Mycelium & Absent, Present \\
\hline Discoloration & Absent, Brown, Black \\
\hline Sclerotium & Absent, Present \\
\hline Fruit Spots & Absent, Dark, Other \\
\hline Roots & Normal, Rotted, Cysts \\
\hline Diseases & Twenty Different Types \\
\hline
\end{tabular}

Using the WEKA machine learning software tool of the University of Waikato [15], the ten best actionable rules have been generated. The most important rules are: a) if Sclerotium is absent then Mycelium is absent; b) if Mycelium is absent then Sclerotium is absent; c) if Discoloration is absent then both Mycelium and Sclerotium are absent; and d) if Leaves are abnormally big then Mycelium is absent.

\section{CoNCLUSION}

The described DSS can be employed in acquiring, processing, and distributing data, information, and rules to treat and protect the agricultural crops.

\section{REFERENCES}

[1] S. Shanthi, R. Aabinaya, V. Akshaya, and S. Gowri, "Agriculture Crop Monitoring Using GSM in WSN", International Journal of Advanced Research in
Computer and Communication Engineering, vol. 5, pp. 606-710, 2016.

[2] A. Kvieses, A. Zacepins, M. Durugun, and S. Tekin, "Application of Wireless Sensor Networks in Precision Apiculture", Proc. 14th International Scientific Conference on Engineering for Rural Development, Jelgava (Latvia) 2015, (pp. 440-445)

[3] P. Gangurde and M. Bhende, "A Review on Precision Agriculture Using Wireless Sensor Networks", International Journal of Engineering Trends and Technology, vol. 23, pp. 426-431, 2015.

[4] J. Polo, G. Hornero, C. Duijneveld, A. Garcia, and O. Casas, "Design of a Low-Cost Wireless Sensor Network with UAV Mobile Node for Agricultural Applications”, Computers and Electronics in Agriculture, vol. 119, pp. 19-32, 2015.

[5] N. Brinis, and L. A. Saidane, "Context Aware Wireless Sensor Network Suitable for Precision Agriculture”, Wireless Sensor Network, vol. 8, pp. 1-12, 2016.

[6] Z. Stamenkovic, S. Randjic, I. Santamaria, U. Pesovic, G. Panic, and S. Tanaskovic, "Advanced Wireless Sensor Nodes and Networks for Agricultural Applications”, Proc. 24th Telecommunications Forum TELFOR, Belgrade (Serbia) 2016, (pp.1-8)

[7] M. O. Farooq and T. Kunz, "Operating Systems for Wireless Sensor Networks: A Survey”, Sensors, vol. 11, pp. 5900-5930, 2011.

[8] D. Markovic, S. Randjic, S. Tanaskovic, and S. Gvozdenac, "Possibility of Monitoring D.v. virgifera Flight by Processing Image of Phero-Traps Using Raspberry Pi Based Devices”, Acta Agriculturae Serbica, vol. 22, pp. 207-217, 2017.

[9] Y. Fang and R. P. Ramasamy, "Current and Prospective Methods for Plant Disease Detection”, Biosensors, vol. 4, pp. 537-561, 2015.

[10]E. Pievanelli, R. Stefanelli, and D. Trinchero, "Microwave-based Leaf Wetness Detection in Agricultural Wireless Sensor Networks”, Proc. IEEE Sensors Applications Symposium, Catania (Italy) 2016, (pp. 1-4)

[11]F. Perez-Cruz, S. Van Vaerenbergh, J. J. MurilloFuentes, M. L. Gredilla, and I. Santamaria, "Gaussian Processes for Nonlinear Signal Processing”, IEEE Signal Processing Magazine, vol. 30, pp. 40-50, 2013.

[12] S. Sladojevic, M. Arsenovic, A. Anderla, D. Culibrk, and D. Stefanovic, "Deep Neural Networks Based Recognition of Plant Diseases by Leaf Image Classification”, Computational Intelligence and Neuroscience, 2016.

[13] I. Goodfellow, Y. Bengio, and A. Courville, “Deep Learning”, MIT Press, 2016.

[14] S. P. Mohanty, D. P. Hughes, and M. Salathé, "Using Deep Learning for Image-Based Plant Disease Detection”, Frontiers in Plant Science, vol. 7, pp. 141901-141910, 2016.

[15] WEKA machine learning software tool, University of Waikato, https://www.cs.waikato.ac.nz/ml/weka/ 